TITLE:

\title{
Some results on open algebraic surfaces of logarithmic Kodaira dimension zero
}

AUTHOR(S):

Kojima, Hideo

\section{CITATION:}

Kojima, Hideo. Some results on open algebraic surfaces of logarithmic Kodaira dimension zero. 代数幾何学シンポジウム記録 2019, 2019: 3-12

ISSUE DATE:

2019

URL:

http://hdl.handle.net/2433/245710

RIGHT: 


\title{
SOME RESULTS ON OPEN ALGEBRAIC SURFACES OF LOGARITHMIC KODAIRA DIMENSION ZERO
}

\author{
HIDEO KOJIMA
}

\section{INTRODUCTION}

This article gives a brief survey of open algebraic surfaces and presents recent results on open algebraic surfaces of logarithmic Kodaira dimension zero in any characteristic.

Section 1 surveys results on open algebraic surfaces. First of all, we recall structure theorems for open algebraic surfaces. Then we recall a minimal model theory of open algebraic surfaces of non-negative logarithmic Kodaira dimension and collect results on open algebraic surfaces of logarithmic Kodaira dimension zero. In Section 2, some results of [17] are introduced. Theorems 2.1 and 2.2 are the main results of this article. In Section 3, we classify the affine plane curves whose complements have logarithmic Kodaira dimension $\leq 0$ by using a result of Ganong [6] and Theorems 2.1 and 2.2 .

Acknowledgements. The author would like to express his gratitude to the organizers Hiraku Kawanoue, Takashi Kishimoto and Shingo Taki for giving him the opportunity to talk at Kinosaki Algebraic Geometry Symposium 2019. The author was supported by JSPS KAKENHI Grant Number JP 17K05198.

\section{KNOWN RESULTS ON OPEN ALGEBRAIC SURFACES}

In this section, we survey results on open algebraic surfaces. Throughout this article, we work over an algebraically closed field $k$.

A reduced effective divisor $D$ on a smooth surface is called an $S N C$ divisor if it has only simple normal crossings. Let $S$ be a smooth open algebraic surface. Then there exist a smooth projective surface $V$ and an SNC-divisor on $V$ such that $V-D(:=V \backslash \operatorname{Supp} D)=S$. The pair $(V, D)$ is called an $S N C$-completion of $S$ and $D$ is called the boundary divisor of $(V, D)$. For a positive integer $n, h^{0}\left(V, n\left(K_{V}+D\right)\right)$ is called the logarithmic n-genus of $S$ and is denoted by $\bar{P}_{n}(S)$. The logarithmic 1-genus of $S$ is also called the logarithmic geometric genus of $S$ and is denoted by $\bar{p}_{g}(S)$. The Iitaka dimension $\kappa\left(V, K_{V}+D\right)$ of $K_{V}+D$ is called the logarithmic Kodaira dimension of $S$ and is denoted by $\bar{\kappa}(S)$. Of course, the numbers $\bar{P}_{n}(S)$ and $\bar{\kappa}(S)$ are independent of the choices of SNC-completions of $S$. We note that $\bar{P}_{n}(S)$ and $\bar{\kappa}(S)$ can be defined when $\operatorname{char}(k)>0$ (see [10]). 
1.1. Some structure theorems. Here we recall some well-known structure theorems for open algebraic surfaces. For more details, we refer to Miyanishi [20] and [22]. Let $S$ be a smooth open algebraic surface.

First of all, we recall some results on surfaces of $\bar{\kappa}=-\infty$.

Theorem 1.1. (Fujita [3], Miyanishi-Sugie [23], Russell [29]) If $S$ is a smooth rational surface of $\bar{\kappa}(S)=-\infty$ with connected boundary at infinity (i.e., the boundary divisor of an SNC-completion of $S$ is connected), then it is affine ruled (i.e., it contains a surface isomorphic to $\mathbb{A}^{1} \times T$, where $T$ is a smooth curve, as a Zariski open subset).

Theorem 1.2. (Miyanishi [21]) If $S$ is a smooth irrational surface of $\bar{\kappa}(S)=-\infty$, then it is affine ruled.

We note that Theorems 1.1 and 1.2 hold true in the case $\operatorname{char}(k)>0$.

Later on, Keel and $\mathrm{M}^{\mathrm{c}}$ Kernan proved the following remarkable result in the case $k=\mathbb{C}$.

Theorem 1.3. (Keel-McKernan [12]) Every open algebraic surface of $\bar{\kappa}=-\infty$ defined over $\mathbb{C}$ is log uniruled.

We next consider the case $\bar{\kappa}(S) \geq 0$. Let $(V, D)$ be an SNC-completion of $S$. Since $\bar{\kappa}(S) \geq 0$, the $\log$ canonical divisor $K_{V}+D$ is pseudo-effective. So we have the Zariski decomposition of $K_{V}+D$ :

$$
K_{V}+D \equiv\left(K_{V}+D\right)^{+}+\left(K_{V}+D\right)^{-},
$$

where $\left(K_{V}+D\right)^{+}\left(\operatorname{resp} .\left(K_{V}+D\right)^{-}\right)$is the nef part of $K_{V}+D$ (resp. the negative part of $\left.K_{V}+D\right)$. Then we have the following result.

Theorem 1.4. (Kawamata [11], Fujita [5] (in any characteristic)) With the same notations and assumptions as above, $\left(K_{V}+D\right)^{+}$is semiample. So we have the following:

(1) $\bar{\kappa}(S)=0 \Longleftrightarrow\left(K_{V}+D\right)^{+} \equiv 0$.

(2) $\bar{\kappa}(S)=1 \Longleftrightarrow\left(\left(K_{V}+D\right)^{+}\right)^{2}=0$ and $\left(K_{V}+D\right)^{+} \not \equiv 0$.

(3) $\bar{\kappa}(S)=2 \Longleftrightarrow\left(\left(K_{V}+D\right)^{+}\right)^{2}>0$.

We consider the following cases.

Case: $\bar{\kappa}(S)=1$. In [11], Kawamata gave a structure theorem for open algebraic surfaces of logarithmic Kodaira dimension one when $\operatorname{char}(k)=$ 0 . The structure theorem is generalized in [15] in any characteristic. Here, we recall briefly the result.

By Theorem 1.4, we know that, for a sufficiently large integer $n$, $\left|n\left(K_{V}+D\right)^{+}\right|$is composed of an irreducible pencil $\Lambda$ without base points. Let $\Phi: V \rightarrow T$ be the fibration associated with $\Lambda$. Then, $\Phi$ is an elliptic fibration, a quasi-elliptic fibration or a $\mathbb{P}^{1}$-fibration. Further, we have a $\log$ canonical bundle formula for the fibration $\Phi$. For more details, we refer to [15].

Case: $\bar{\kappa}(S)=2$. In this case, the log canonical ring of $S$ is finitely generated over $k$ by Theorem 1.4. Of course, the classification of such surfaces is not fully understood. 
1.2. Construction of strongly minimal models. There are several minimal model theories of open algebraic surfaces. Here, we use the one by Tsunoda [30]. The results of $[30, \S 1]$ are generalized in MiyanishiTsunoda [24, Chapter 1] (see also [22, Chapter 2]). Here we use the result of [30] because the theory given in [24, Chapter 1] needs graph theoretic arguments.

Lemma 1.5. (cf. [30, Theorem 1.3]) With the same notations as above, assume that $\bar{\kappa}(S) \geq 0$. Let $f: V \rightarrow W$ be a successive contractions of $(-1)$-curves in $\operatorname{Supp}\left(K_{V}+D\right)^{-}$and set $C=f_{*}(D)$. Then we have:

(1) $C$ is an $S N C$-divisor.

(2) $\bar{P}_{n}(W-C)=\bar{P}_{n}(X-B)$ for any positive integer $n$. In particular, $\bar{\kappa}(W-C)=\bar{\kappa}(X-B)=0$.

(3) $f_{*}\left(\left(K_{V}+D\right)^{+}\right)=\left(K_{W}+C\right)^{+}$.

(4) $\left(K_{W}+C\right)^{-} \leq C$ and each connected component of $\operatorname{Supp}\left(K_{W}+C\right)^{-}$ can be contracted to a KLT singular point.

The SNC-pair $(W, C)$ is called an almost minimal model of $(X, B)$.

From now on, we mainly consider open algebraic surfaces of logarithmic Kodaira dimension zero. In order to state some results of such surfaces, we introduce how to construct their strongly minimal models.

Assume that $\bar{\kappa}(S)=0$. Then $\left(K_{V}+D\right)^{+} \equiv 0$ by Theorem 1.4 and so $\left(K_{W}+C\right)^{+} \equiv 0$. Set $C^{\#}:=C-\left(K_{W}+C\right)^{-}$and $C^{\prime}:=C-\left\lfloor C^{\#}\right\rfloor$. Let $\pi: W \rightarrow \bar{W}$ be the contraction of $\operatorname{Supp} C^{\prime}$. Here $\bar{W}$ is a normal projective surface with only KLT singular points by (4) of Lemma 1.5. So we can run a $\log$ MMP for the surface $\bar{W}$. Let $\bar{g}: \bar{W} \rightarrow \bar{X}$ be the corresponding birational map. Let $\pi^{\prime}: X \rightarrow \bar{X}$ be the minimal resolution of $\bar{X}$. Then there exists a birational morphism $g: W \rightarrow X$ such that the following diagram commutes.

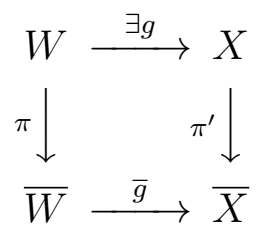

Set $B:=g_{*}(C)$. We note that the divisor $B$ may not be an SNCdivisor. We call the pair $(X, B)$ a strongly minimal model of $(X, B)$. We often call the surface $W-B$ (resp. $X-B$ ) a strongly minimal model (resp. an almost minimal model) of $S$. Moreover, the surface $S$ is said to be strongly minimal (resp. almost minimal) if there exists a strongly minimal model $(X, B)$ (resp. an almost minimal model $(W, C)$ ) of a SNCcompletion of $S$ such that $S=X-B$ (resp. $S=W-C)$.

1.3. Some known results on open algebraic surfaces of $\bar{\kappa}=0$. We recall some results on open algebraic surfaces of $\bar{\kappa}=0$. This subsection consists of three parts.

(I) By the results of Tsunoda [30] and Blache [2], we have the following result. 
Theorem 1.6. Assume that $\operatorname{char}(k)=0$. Let $S$ be a smooth open algebraic surface of $\bar{\kappa}(S)=0$ and set $I(S):=\min \left\{n \in \mathbb{N} \mid \bar{P}_{n}(S)>0\right\}$. Then we have:

(1) $1 \leq I(S) \leq 21$.

(2) Let $(V, D)$ be an $S N C$-completion of $D$. If $D$ is connected or the intersection matrix of $D$ is not negative definite, then $1 \leq I(S) \leq$ 6 .

(II) A log Enriques surface is, by definition, a normal projective rational surface with only KLT singular points and with numerically trivial canonical divisor. The smooth part of a log Enriques surfaces is an interesting example of an almost minimal open rational surface of $\bar{\kappa}=\bar{p}_{g}=0$. Log Enriques surfaces have been studied by many mathematicians (when $\operatorname{char}(k)=0)$. For more details, see Blache [2], Kudryavtsev [18] [19], Oguiso-Zhang [26] [27] [28], Zhang [32] [33] [34] [35] [36], etc.

(III) In order to study open algebraic surfaces of $\bar{\kappa}=0$, it is important to classify their strongly minimal models. We have some partial classification results for the strongly minimal models. We recall some of them without explicit classification. Let $S,(V, D),(W, C)$ and $(X, B)$ be the same as above.

(III-1) Since $\bar{\kappa}(S)=0$, the Kodaira dimension $\kappa(V)$ of $V \leq 0$. If $\kappa(V)=0$, then $C^{\#}=0$ and $K_{W} \equiv 0$. So $W$ is a minimal surface of $\bar{\kappa}(V)=0$ and each connected component of $C$ can be contracted to a canonical singular point.

(III-2) Suppose that $\kappa(V)=-\infty$. Then strongly minimal smooth open algebraic surfaces are classified in the following cases, where $S$ is a strongly minimal and $(V, D)$ is an SNC-completion of $S$.

- $S$ is an irrational ruled surface. (Iitaka [7] [8] (when $\operatorname{char}(k)=0)$, K. [16] (in any characteristic).)

- $S$ is a rational surface, $D$ is connected and the intersection matrix of $D$ is not negative definite. (K. [13] (in any characteristic). See also Fujita [4].)

- $S$ is a rational surface, $\bar{p}_{g}(S)=1$ and $\operatorname{char}(k)=0$. (Iitaka [7] [8] (partially), Zhang [31].)

- $S$ is a rational surface, $\bar{p}_{g}(S)=0, \bar{P}_{2}(S)=1$, the intersection matrix of $D$ is not negative definite and $\operatorname{char}(k)=0$. (K. [14].)

\section{Open Algebraic surfaces of $\bar{\kappa}=0$ With LOW DefeCts}

We work over an algebraically closed field $k$. Let $S$ be a smooth open algebraic surface and let $(V, D)$ be an SNC-completion of $S$.

In [25], Nakayama defined the defect of a pair of a normal Moishezon surface and a reduced divisor on it. The defect of $(V, D)$ is $\rho(V)+2-\# D$, where $\rho(V)$ is the Picard number of $V$ and $\# D$ is the number of all irreducible components in $\operatorname{Supp} D$. We then define the number $\delta(S)$ as $\delta(S)=\rho(V)+2-\# D$, that is the defect of $(V, D)$. The number $\delta(S)$ does not depend on the choices of the SNC-completions of $S$. 
For a non-negative integer $r$, set $\mathbb{A}_{(r)}^{1}=\mathbb{A}_{k}^{1}-\{r$ points $\}$. We consider a smooth affine surface $S_{r, s}=\mathbb{A}_{(r)}^{1} \times \mathbb{A}_{(s)}^{1}$ for two non-negative integers $r$ and $s$. It is clear that $\delta\left(S_{r, s}\right)=2-(r+s)$. Furthermore, we easily see that $\bar{\kappa}\left(S_{r, s}\right)=-\infty\left(\operatorname{resp} . \bar{\kappa}\left(S_{r, s}\right)=0, \bar{\kappa}\left(S_{r, s}\right)=1, \bar{\kappa}\left(S_{r, s}\right)=2\right)$ if $\min \{r, s\}=0$ (resp. $r=s=1, \min \{r, s\}=1$ and $\max \{r, s\} \geq 2$, $\min \{r, s\} \geq 2)$. This shows that, for $\ell \in\{-\infty, 1,2\}$, there does not exist lower bound for the set of numbers $\delta(S)$ of the smooth open algebraic surfaces $S$ of $\bar{\kappa}(S)=\ell$.

In this section, some results of [17] are introduced. In [17], the author considers the following problems motivating the recent work on classifications of the almost toric surfaces and the half-toric surfaces by Nakayama [25].

Problem 1. Does there exist $\min \{\delta(S) \mid S$ is a smooth open algebraic surface of $\bar{\kappa}(S)=0\}$ ? If so, give the minimal number.

Problem 2. Classify the open algebraic surfaces $S$ of $\bar{\kappa}(S)=0$ with low $\delta(S)$.

The main results of this article are as follows:

Theorem 2.1. (cf. [17, Theorem 1.1]) Let $S$ be a smooth open algebraic surface of $\bar{\kappa}(S)=0$. Then the following assertions hold true:

(1) $\delta(S) \geq 0$.

(2) $\delta(S)=0$ if and only if $S$ is isomorphic to $\mathbb{G}_{m}^{2}$ minus $n$ points $(n \geq 0)$, where $\mathbb{G}_{m}^{2}$ is the two dimensional algebraic torus over $k$.

Theorem 2.2. (cf. [17, Theorem 1.2]) Let $S$ be a smooth open algebraic surface of $\bar{\kappa}(S)=\bar{p}_{g}(S)=0$. Then the following assertions hold true:

(1) $\delta(S) \geq 1$

(2) $\delta(S)=1$ if and only if $S$ is a surface constructed in Example 2.3.

Example 2.3. Let $V_{0}=\Sigma_{1}$ be the Hirzebruch surface of degree one, let $M_{1}$ be the minimal section on $V_{0}$ and let $\ell$ be a fiber of the ruling $\pi$ on $V_{0}$. Let $C^{\prime}$ be an irreducible curve with $C^{\prime} \sim 2 M_{1}+2 \ell$. Since $\left.\pi\right|_{C^{\prime}}: C^{\prime} \rightarrow \mathbb{P}^{1}$ is a finite morphism of degree two, there exist at least two fibers, say $\ell_{1}$ and $\ell_{2}$, of $\pi$ such that $\# \ell_{i} \cap C^{\prime}=1$ for $i=1$, 2. Let $\mu_{0}: V_{1} \rightarrow V_{0}$ be the blowing-up with centers $\ell_{1} \cap C^{\prime}$ and $\ell_{2} \cap C^{\prime}$ and set $E_{i}:=\mu^{-1}\left(\ell_{i} \cap C^{\prime}\right)(i=1,2)$. Let $\mu_{1}: V_{2} \rightarrow V_{1}$ be the blowing-up with centers $Q_{1}:=E_{1} \cap \mu_{0}^{\prime}\left(\ell_{1}\right)$ and $Q_{2}:=E_{2} \cap \mu_{0}^{\prime}\left(\ell_{2}\right)$ and set

$$
D^{(0)}=\mu_{1}^{-1}\left(Q_{1}\right)+\mu_{1}^{-1}\left(Q_{2}\right)+\mu_{1}^{\prime}\left(E_{1}+E_{2}+\mu_{0}^{\prime}\left(C^{\prime}+\ell_{1}+\ell_{2}\right)\right) .
$$

Then $\bar{\kappa}\left(V_{2}-D^{(0)}\right)=\bar{p}_{g}\left(V_{2}-D^{(0)}\right)=0$ and $\bar{P}_{2}\left(V_{2}-D^{(0)}\right)=1$. The surface $V_{2}-D^{(0)}$ is called $H[-1,0,-1]$ in $[4,(8.5)]$ (see also [13, Example 2.1]).

Assume further that $\left.\pi\right|_{C^{\prime}}: C^{\prime} \rightarrow \mathbb{P}^{1}$ is not separable. Then $\operatorname{char}(k)=2$ and $\left.\pi\right|_{C^{\prime}}$ is purely inseparable. Let $P_{1}, \ldots, P_{r}(r \geq 0)$ be points on $\mu_{1}^{\prime}\left(\mu_{0}^{\prime}\left(C^{\prime}\right)\right) \backslash\left\{\mu_{1}^{-1}\left(Q_{1}\right) \cup \mu_{1}^{-1}\left(Q_{2}\right)\right\}$ and let $F_{i}(1 \leq i \leq r)$ be the fiber of the ruling $\pi^{\prime}=\pi \circ \mu_{0} \circ \mu_{1}: V_{2} \rightarrow \mathbb{P}^{1}$ passing through $P_{i}$. Let $\mu_{2}: V_{3} \rightarrow V_{2}$ be a composite of blowing-ups over points $P_{1}, \ldots, P_{r}$ such that the fiber 
$\mu_{2}^{*}\left(F_{i}\right)(1 \leq i \leq r)$ of $\pi^{\prime} \circ \mu_{2}: V_{3} \rightarrow \mathbb{P}^{1}$ has the dual graph in Figure 1, where $\mu_{2}^{*}\left(F_{i}\right)=2\left(G_{1}+D_{1}^{i}+\cdots+D_{s_{i}-2}^{i}\right)+D_{s_{i}-1}^{i}+D_{s_{i}}^{i}$ and $s_{i} \geq 2$. Set $V=V_{3}$ and $D=\mu_{2}^{\prime}\left(D^{(0)}\right)+\sum_{i=1}^{r}\left(\sum_{j=1}^{s_{i}} D_{j}^{i}\right)$, where $V=V_{2}$ and $D=D^{(0)}$ if $r=0$ or $\left.\pi\right|_{C^{\prime}}$ is separable. Then $\bar{\kappa}(V-D)=\bar{\kappa}\left(V_{2}-D^{(0)}\right)=0$, $\bar{p}_{g}(V-D)=\bar{p}_{g}\left(V_{2}-D^{(0)}\right)=0$ and $\bar{P}_{2}(V-D)=\bar{P}_{2}\left(V_{2}-D^{(0)}\right)=1$.

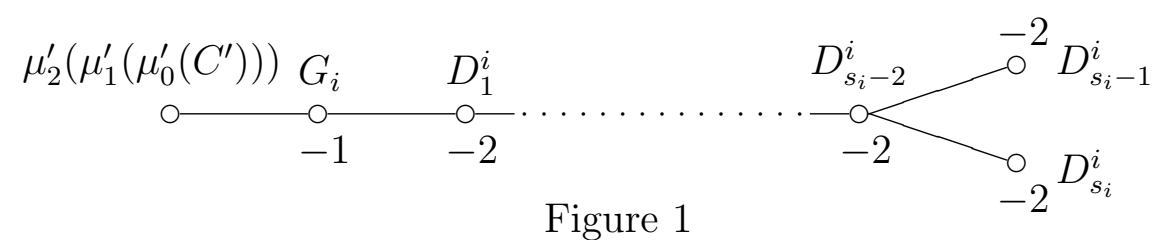

We outline the proofs of Theorems 2.1 and 2.2 in [17].

Let $S$ be a smooth open algebraic surface of $\bar{\kappa}(S)=0$ and let $(V, D)$ be an SNC-completion of $S$. Let $(X, B)$ be a strongly minimal model of $(V, D)$ (cf. 1.2 of $\S 1$ ). Then we have a birational morphism $f: V \rightarrow X$ such that $f_{*}(D)=B$. The following lemma can be proved easily.

Lemma 2.4. With the same notations and assumptions as above, set $\tilde{S}:=X-B$. Then we have:

(1) $\delta(S) \geq \delta(\tilde{S})$

(2) $\delta(S)=\delta(\tilde{S})$ if and only if $S=\tilde{S}-\{n$ points $\}$ for some integer $n \geq 0$.

(3) If $S$ is the smooth part of a normal affine surface, then $\delta(S)=$ $\delta(\tilde{S}) \Longleftrightarrow S=\tilde{S}$.

Case 1: $S$ is not a rational surface. In this case, by using results of [16], we have the following lemma. See $[17, \S 3]$ for its proof.

Lemma 2.5. Suppose that $S$ is not a rational surface. Then the following assertions hold true.

(1) If $S$ is not birationally ruled, then $\delta(S) \geq 3$.

(2) If $S$ is birationally ruled, then $\delta(S) \geq 2$. Moreover, $\delta(S)=2$ if and only if $S=V-D-\{n$ points $\}$, where $n \geq 0, V=\mathbb{P}_{E}\left(\mathcal{O}_{E} \oplus \mathcal{L}\right)$, where $E$ is an elliptic curve and $\mathcal{L} \in \operatorname{Pic}(E)$, and $D=D_{1}+D_{2}$ is a sum of two disjoint sections $D_{1}$ and $D_{2}$ of the ruling $\pi: V \rightarrow E$.

Case 2: $S$ is a rational surface. We use the notations in Lemma 2.4. Let $\pi^{\prime}: X \rightarrow \bar{X}$ be the birational morphism defined in 1.2 of $\S 1$. The divisor $B$ may not be an SNC-divisor. In fact, we know that the divisor $B$ is an SNC-divisor if $\delta(S) \leq 1$.

From now on, we assume further that $\delta(S) \leq 1$. Then $\delta(\tilde{S}) \leq \delta(S) \leq 1$ by Lemma 2.4. So the intersection matrix of $B$ is not negative definite by the Hodge index theorem. In particular, $\bar{B}:=\pi^{\prime}(B) \neq 0$.

By the construction of a strongly minimal model of $S$, we see that $K_{\bar{X}}+\bar{B}=\bar{g}\left(K_{\bar{W}}+\pi(C)\right) \equiv 0$, where $\bar{g}: \bar{W} \rightarrow \bar{X}$ is the birational morphism defined in 1.2 of $\S 1$. So $K_{\bar{X}}$ is not nef since $\bar{B} \neq 0$. Hence, we obtain the following lemma. 
Lemma 2.6. With the same notations and assumptions as above, one of the following two cases takes place.

(I) There exists a $\mathbb{P}^{1}$-fibration $p: \bar{X} \rightarrow \mathbb{P}^{1}$ such that every fiber of $p$ is irreducible.

(II) $-K_{\bar{X}}$ is ample and $\rho(\bar{X})=1$. (In this case, $\bar{X}$ is a log del Pezzo surface of rank one.)

We consider the following cases separately.

Case (I) in Lemma 2.6. Then the morphism $p \circ \pi^{\prime}$ is a $\mathbb{P}^{1}$-fibration from $X$ onto $\mathbb{P}^{1}$. By using this $\mathbb{P}^{1}$-fibration, we can determine the pair $(X, B)$ when either $\delta(\tilde{S}) \leq 0$ or $\delta(\tilde{S})=1$ and $\bar{p}_{g}(S)=0$. Hence the assertions of Theorems 2.1 and 2.2 are verified in this case. For more details, see [17, $\S \S 4$ and 5].

Case (II) in Lemma 2.6. In this case, since $\rho(X)=\rho(\bar{X})+\# B-\# \bar{B}$ and $\rho(\bar{X})=1$, we have $\delta(\tilde{S})=\rho(X)+2-\# B=1-\# \bar{B}$. So, $\# \bar{B}=$ $1-\delta(\tilde{S}) \geq 2$. We can determine the pair $(X, B)$ when either $\delta(\tilde{S})=0$ or $\delta(\tilde{S})=1$ and $\bar{p}_{g}(S)=0$. More precisely, we have the following lemma.

Lemma 2.7. With the same notations and assumptions as above, the following assertions hold true:

(1) $\delta(\tilde{S}) \geq 0$. Moreover, if $\delta(\tilde{S})=0$, then $\tilde{S} \cong \mathbb{G}_{m}^{2}$.

(2) If $\delta(\tilde{S})=1$, then $\bar{p}_{g}(S)=1$.

See $[17, \S \S 4$ and 5] for its proof. To prove (2) of Lemma 2.7 is the most difficult part in the proofs of Theorems 2.1 and 2.2 in [17].

Theorems 2.1 and 2.2 are thus proved.

\section{Affine plane CurVes Whose Complements have LOGARITHMIC KODAIRA DIMENSION $\leq 0$}

We work over an algebraically closed field $k$. When $\operatorname{char}(k)=0$, a list of the affine plane curves whose complements have logarithmic Kodaira dimension $\leq 1$ is given in Iitaka [9]. See also Aoki [1]. In this section, we give a classification of the affine plane curves whose complements have logarithmic Kodaira dimension $\leq 0$ in any characteristic. In fact, the results are the same as those in the characteristic zero case.

Let $k^{[2]}=k[x, y]$ be the polynomial ring in two variables over $k$. Let $C=V(f) \subset \mathbb{A}_{k}^{2}=$ Spec $k^{[2]}$ be an affine plane curve with defining equation $f \in k^{[2]}$ and set $S:=\mathbb{A}^{2}-C$. Let $C=\cup_{i=1}^{r} C_{i}$ be the decomposition of $C$ into irreducible components.

We first consider the case $\bar{\kappa}(S)=-\infty$. Since $\delta(S)=2-r \leq 1$, we have a result stronger than Theorem 1.1. See [17, Lemma 2.8]. By using this result, we know that each irreducible component $C_{i}$ of $C$ is smooth and isomorphic to $\mathbb{A}^{1}$. Then we infer from $\left[6\right.$, Theorem 2.4] that each $C_{i}$ is a coordinate line. Therefore, we have the following proposition. 
Proposition 3.1. (cf. [17, Proposition 6.1]) With the same notations and assumptions as above, assume further that $\bar{\kappa}(S)=-\infty$. Then there exists a system $\{x, y\}$ of variables of $k^{[2]}$ and distinct $r$ elements $\alpha_{1}, \ldots, \alpha_{r} \in k$ such that $f=\prod_{i=1}^{r}\left(x-\alpha_{i}\right)$.

We next consider the case $\bar{\kappa}(S)=0$.

Lemma 3.2. (cf. [17, Lemma 6.2]) With the same notations and assumptions as above, the following assertions hold true.

(1) $r \leq 2$. Moreover, $r=2$ if and only if $S \cong \mathbb{G}_{m}^{2}$.

(2) $\bar{p}_{g}(S)=1$.

Proof. (1) Since $\delta(S)=2-r$ and $S$ is affine, the assertion (1) follows from Theorem 2.1 .

(2) If $r=2$, then $\bar{p}_{g}(S)=\bar{p}_{g}\left(\mathbb{G}_{m}^{2}\right)=1$. Assume that $r=1$. If $\bar{p}_{g}(S)=$ 0 , then we infer from Theorem 2.2 that $S$ is the surface $H[-1,0,-1]$, here we note that $S$ is smooth and affine. Then $\operatorname{Pic}(S) \cong \mathbb{Z} / 2 \mathbb{Z}$ by $[13$, p. 949$]$ (see also $[4,(8.5)]$ ). This is a contradiction because the coordinate ring of $S=\mathbb{A}^{2}-C$ is a UFD. Therefore, $\bar{p}_{g}(S)=1$.

The following lemma can be proved by the construction of a strongly minimal model of $S$. We omit its proof.

Lemma 3.3. (cf. [17, Lemma 6.3]) Assume that $S$ is not strongly minimal in the sense of $\S 1$. Then $S$ contains an affine line $L$ such that $\bar{\kappa}(S-L)=\bar{\kappa}(S)=0$. In particular, $r=1$.

In Lemma $3.3, S-L \cong \mathbb{G}_{m}^{2}$ by Lemma $3.2(1)$.

The following lemma can be proved by the classification of strongly minimal smooth affine surfaces of $\bar{\kappa}=0$ in [13]. We omit its proof.

Lemma 3.4. (cf. [17, Lemma 6.4]) Assume that $S$ is strongly minimal in the sense of $\S 1$. Then either $S \cong \mathbb{G}_{m}^{2}$ or $S \cong V(x y-1)$ for a suitable system $\{x, y\}$ of variables of $k^{[2]}$. If the latter case occurs, then $S$ contains an affine line $L$ such that $S-L \cong \mathbb{G}_{m}^{2}$.

We determine the defining equation of $C$. We use the same notations as above. If $r \geq 2$, then $r=2$ and $S \cong \mathbb{G}_{m}^{2}$ by Lemma 3.2 (1). If $r=1$, then $S$ contains an affine line $L$ such that $S-L \cong \mathbb{G}_{m}^{2}$. Of course, $L$ is closed in $\mathbb{A}_{k}^{2}$. In order to determine the defining equation of $C$, it is sufficient to consider the case $r=2$ only.

Lemma 3.5. (cf. [17, Lemma 6.5]) With the same notations and assumptions as above, assume that $r=2$. Then at least one of $\bar{\kappa}\left(\mathbb{A}_{k}^{2}-C_{1}\right)$ and $\bar{\kappa}\left(\mathbb{A}_{k}^{2}-C_{2}\right)$ equals $-\infty$.

See [17, Lemma 6.5] for its proof.

The following theorem is the main result of this section.

Theorem 3.6. (cf. [17, Theorem 6.6]) Let $C=C_{1} \cup C_{2}$ be an affine plane curve with two irreducible components $C_{1}$ and $C_{2}$. Assume that 
$S=\mathbb{A}_{k}^{2}-C \cong \mathbb{G}_{m}^{2}$ and $\bar{\kappa}\left(\mathbb{A}_{k}^{2}-C_{2}\right)=-\infty$. Then there exists a system $\{x, y\}$ of variables of $k^{[2]}$ such that

$$
C_{1}=V\left(x^{m} y+p(x)\right), \quad C_{2}=V(x),
$$

where $m \in \mathbb{Z}_{\geq 0}$ and $p(x) \in k[x]$ is a polynomial in $k[x]$ such that $p(0) \neq 0$ and $\operatorname{deg} p(x)<m($ resp. $p(x)=0)$ if $m>0$ (resp. $m=0)$. Furthermore,

$$
\bar{\kappa}\left(\mathbb{A}_{k}^{2}-C_{1}\right)= \begin{cases}0 & \text { if } m \geq 1, \\ -\infty & \text { if } m=0 .\end{cases}
$$

By Proposition 3.1, $C_{2}=V(x)$ for a system $\{x, y\}$ of variables of $k^{[2]}$. Then, by using the argument as in [23, p. 350], we can determine the defining equation of $C$. For more details, see [17, Theorem 6.6]. Theorem 3.6 is thus verified.

\section{REFERENCES}

[1] H. Aoki, Étale endomorphisms of smooth affine surfaces, J. Algebra, 226 (2000), $15-52$.

[2] R. Blache, The structure of l.c. surfaces of Kodaira dimension zero, I, J. Algebraic Geom., 4 (1995), 137-179.

[3] T. Fujita, On Zariski problem, Proc. Japan Acad. Ser. A Math. Sci., 55 (1979), 106-110.

[4] T. Fujita, On the topology of non-complete algebraic surfaces, J. Fac. Sci. Univ. Tokyo, 29 (1982), 503-566.

[5] T. Fujita, Fractionally logarithmic canonical rings of algebraic surfaces, J. Fac. Sci. Univ. Tokyo, 30 (1984), 685-696.

[6] R. Ganong, Kodaira dimension of embeddings of the line in the plane, J. Math. Kyoto Univ., 25 (1985), 649-657.

[7] S. Iitaka, A numerical criterion of quasi-abelian surfaces, Nagoya Math. J., 73 (1979), 99-115.

[8] S. Iitaka, On logarithmic K3 surfaces, Osaka Math. J., 16 (1979), 675-705.

[9] S. Iitaka, Birational geometry and logarithmic forms, Recent progress of algebraic geometry in Japan, 1-27, North-Holland Math. Stud., 73, North-Holland, Amsterdam, 1983.

[10] T. Kambayashi, On Fujita's strong cancellation theorem for the affine plane, J. Fac. Sci. Univ. Tokyo, 27 (1980), 535-548.

[11] Y. Kawamata, On the classification of non-complete algebraic surfaces, In: Algebraic geometry (Proc. Summer Meeting, Univ. Copenhagen, Copenhagen, 1978), Lecture Notes in Mathematics 732, Springer, Berlin, 1979, pp. 215-232.

[12] S. Keel and J. McKernan, Rational curves on quasi-projective surfaces, Mem. Amer. Math. Soc., 669 (1999).

[13] H. Kojima, Open surfaces of logarithmic Kodaira dimension zero in arbitrary characteristic, J. Math. Soc. Japan, 53 (2001), 933-955.

[14] H. Kojima, Open algebraic surfaces with $\bar{\kappa}=\bar{p}_{g}=0$ and $\bar{P}_{2}>0$, Osaka J. Math., 48 (2011), 1063-1084.

[15] H. Kojima, Open algebraic surfaces of logarithmic Kodaira dimension one, In: Affine Algebraic Geometry (eds. K. Masuda, H.Kojima and T. Kishimoto), World Scientific, 2013, pp. 135-159.

[16] H. Kojima, Irrational open surfaces of non-negative logarithmic Kodaira dimension, Adv. Stud. Pure Math., 75 (2017), 189-206.

[17] H. Kojima, Some results on open algebraic surfaces of logarithmic Kodaira dimension zero, J. Algebra, 547 (2020), 238-261. 
[18] S. A. Kudryavtsev, Classification of logarithmic Enriques surfaces with $\delta=2$, Math. Notes, 72 (2002), 660-666.

[19] S. A. Kudryavtsev, Classification of Enriques $\log$ surfaces with $\delta=1$, Math. Notes, 76 (2004), 81-89.

[20] M. Miyanishi, Non-complete algebraic surfaces, Lecture Notes in Mathematics, No. 857, Berlin-Heiderberg-New York, Springer, 1981.

[21] M. Miyanishi, On affine-ruled irrational surfaces, Invent. Math., 70 (1982), 2743.

[22] M. Miyanishi, Open algebraic surfaces, CRM Monograph Series 12, American Mathematical Society, Providence, RI, 2001.

[23] M. Miyanishi and T. Sugie, Affine surfaces containing cylinderlike open sets, J. Math. Kyoto Univ., 17 (1980), 11-42.

[24] M. Miyanishi and S. Tsunoda, Non-complete algebraic surfaces with logarithmic Kodaira dimension $-\infty$ and with non-connected boundaries at infinity, Japan. J. Math., 10 (1984), 195-242.

[25] N. Nakayama, A variant of Shokurov's criterion of toric surfaces, Adv. Stud. Pure Math., 75 (2107), 287-392.

[26] K. Oguiso and D.-Q. Zhang, On the most algebraic $K 3$ surfaces and the most extremal log Enriques surfaces, Amer. J. Math., 118 (1996), 1277-1297.

[27] K. Oguiso and D.-Q. Zhang, On extremal log Enriques surfaces, II, Tôhoku Math. J., 50 (1998), 419-436.

[28] K. Oguiso and D.-Q. Zhang, On the complete classification of extremal log Enriques surfaces, Math. Z., 231 (1999), 23-50.

[29] P. Russell, On affine ruled rational surfaces, Math. Ann., 255 (1981), 287-302.

[30] S. Tsunoda, Structure of open algebraic surfaces, I, J. Math. Kyoto Univ., 23 (1983), 95-125.

[31] D.-Q. Zhang, On Iitaka surfaces, Osaka J. Math., 24 (1987), 417-460.

[32] D.-Q. Zhang, Logarithmic Enriques surfaces, J. Math. Kyoto Univ., 31 (1991), 419-466.

[33] D.-Q. Zhang, Logarithmic Enriques surfaces, II, J. Math. Kyoto Univ., 33 (1993), 183-193.

[34] D.-Q. Zhang, Normal algebraic surfaces with trivial tricanonical divisors, Publ. Res. Inst. Math. Sci., 33 (1997), 427-442.

[35] D.-Q. Zhang, Quotients of $K 3$ surfaces modulo involutions, Japan. J. Math., 24 (1998), 335-366.

[36] D.-Q. Zhang, Normal algebraic surfaces with trivial two or four times of the canonical divisors, Internat. J. Math. 9 (1998), 377-406.

(H. Kojima) Department of Mathematics, Faculty of Science, Niigata UNIVERsity, 8050 IKARASHININOCHO, Nishi-KU, NiIgATA 950-2181, JAPAN

Email address: kojima@math.sc.niigata-u.ac.jp 